\title{
A New PMU-Based Fault Detection/Location Technique for Transmission Lines With Consideration of Arcing Fault Discrimination-Part II: Performance Evaluation
}

\author{
Ying-Hong Lin, Member, IEEE, Chih-Wen Liu, Senior Member, IEEE, and Ching-Shan Chen, Student Member, IEEE
}

\begin{abstract}
The theory and algorithms of the proposed technique have been presented in Part I of this two-paper set. In Part II of this two-paper set, the proposed technique is evaluated by considerable simulation cases simulated by the Matlab/Power system Blockset simulator. For the proposed fault detector, the trip time achieved can be up to $3.25 \mathrm{~ms}$ and the average value of trip times is about $8 \mathrm{~ms}$ for both permanent and arcing faults on transmission lines. For the proposed fault locator, the accuracy can be up to $\mathbf{9 9 . 9 9 \%}$ and the error does not exceed $\mathbf{0 . 4 5 \%}$. Moreover, the proposed arcing fault discriminator can discriminate between arcing and permanent faults within four cycles after fault inception. It has proven to be an effective tool to block reclosing on the permanent faults in the computer simulations. The simulation results also demonstrate that the presented extended discrete Fourier transform algorithm eliminates effectively the error caused by exponentially decaying dc offset on fundamental and harmonic phasor computations. Finally, a test case using the real-life measured data proves the feasibility of the proposed technique.
\end{abstract}

Index Terms-Arcing fault discrimination, extended discrete Fourier transform (EDFT), fault detection, fault location, phasor-measurement unit (PMU), transmission-line digital protection.

\section{INTRODUCTION}

$\mathbf{I}_{\mathrm{a}}^{\mathrm{N}}$ $\mathrm{N}$ POWER SYSTEMS, transmission-line protection is a well-known problem that has been studied for a long time [1]-[6]. One of the most attractive approaches on transmission-line protection is the phasor-measurement unit (PMU)-based protection scheme [4]-[6]. In our previous paper [4], [5], the laboratory and field tests on the prototypes of PMU have been reported. The timing error of the implemented PMUs remains within $1 \mu \mathrm{s}$ (equivalent to $0.0216^{\circ}$ phase difference at $60 \mathrm{~Hz}$ ) and certifies the synchronism for the application on PMU-based transmission-line protection. In Part I of this two-paper set, the theory and algorithms of the proposed fault detection/location technique with consideration of arcing fault discrimination have been presented. Furthermore, in Part II of

Manuscript received January 6, 2003; revised May 20, 2003. This work was supported by the National Science Council of the Republic of China under Contract NSC91-2213-E-309-006.

Y.-H. Lin is with the Department of Engineering and Management of Advanced Technology, Chang Jung Christian University, Tainan 711, Taiwan, R.O.C. (e-mail: inhon@mail.cju.edu.tw).

C.-W. Liu and C.-S. Chen are with the Department of Electrical Engineering, National Taiwan University, Taipei 106, Taiwan, R.O.C. (e-mail: cwliu@cc.ee.ntu.edu.tw; d89921019@ms89.ntu.edu.tw).

Digital Object Identifier 10.1109/TPWRD.2004.835276 this two-paper set, the proposed technique is evaluated by considerable simulation cases simulated by Matlab/Power system Blockset simulator and a test case using real-life measured data.

Part II of this two-paper set is organized into four sections. Section I is the introduction. In Section II, the performance evaluation of the extended discrete Fourier transform (EDFT) algorithm is demonstrated through phasor computations on the test signals. In Section III, the performance evaluation of proposed fault detector/locator and arcing fault discriminator is demonstrated. Finally, the conclusions are given in Section IV.

\section{Performance Evaluation of EDFT Algorithm}

In this section, a test signal $\mathrm{x}(\mathrm{t})$ given in (1) is utilized to evaluate the EDFT algorithm presented in Part I of this twopaper set. Furthermore, the performance of DFT-mimic filter configuration [1] and discrete Fourier transform (DFT) is also demonstrated. The implementation of a digital mimic filter is presented in [7].

First, the evaluation is performed under the scenario that both the time constant $\tau_{s}$ in (1) and time constant $\tau_{m}$ in digital mimic filter are set as $2 \times T$ ( 2 times the period of fundamental component)

$$
\begin{aligned}
x(t)= & 100 \cos (\omega t)+5 \cos \left(3 \omega t+\frac{\pi}{3}\right) \\
& +1.5 \cos \left(5 \omega t+\frac{\pi}{5}\right)+0.5 \cos \left(7 \omega t+\frac{\pi}{7}\right) \\
& +0.15 \cos \left(9 \omega t+\frac{\pi}{9}\right)+100 \exp \left(-\frac{t}{\tau_{s}}\right), \\
\omega= & 2 \pi \times 60, \mathrm{~T}=\frac{1}{60} .
\end{aligned}
$$

To save the space, only the fundamental phasor computation and the 9th harmonic phasor computation are demonstrated in Figs. 1 and 2, respectively. For phasor computation by DFTmimic filter configuration, the computed magnitude of the 9th harmonic phasor is magnified due to the frequency response of a digital mimic filter. However, a correction multiplier can be utilized to remove this offset.

Taking observation on Fig. 1 and 2, the accuracy of phasor computations by EDFT and DFT-mimic filter configuration with the correct setting of time constant is not affected by exponentially decaying dc offset. But the accuracy of phasor computation by DFT is corrupted largely due to the appearance of exponentially decaying dc offset. 


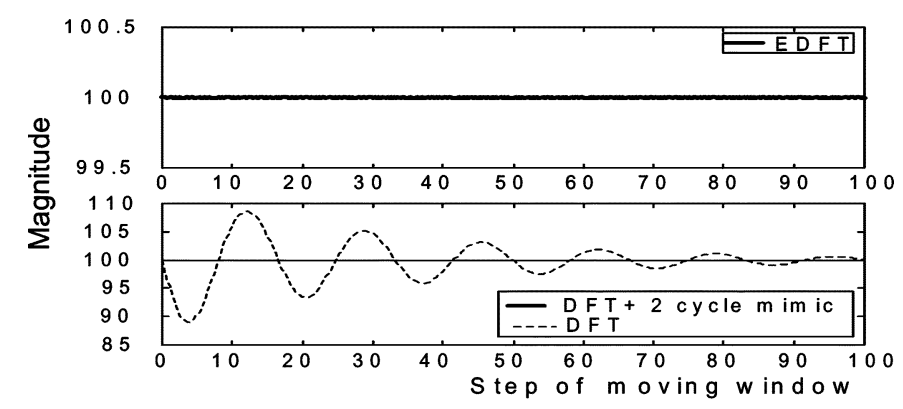

Fig. 1. Computations of the fundamental phasor $\left(\tau_{s}=\tau_{m}=2 \times T\right)$.

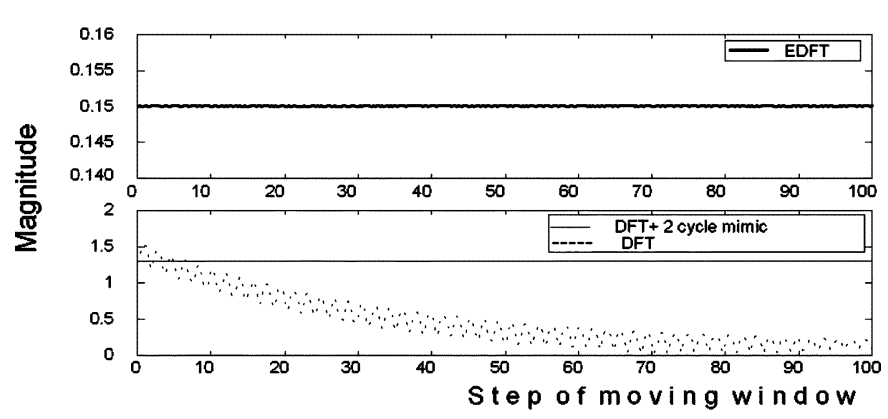

Fig. 2. Computations of the 9 th harmonic phasor $\left(\tau_{s}=\tau_{m}=2 \times T\right)$.

In practice, the time constant in a measured signal is uncertain when a fault occurs on transmission lines. So we change deliberately the time constant $\tau_{s}$ to $0.5 \times \mathrm{T}$. But the time constant $\tau_{m}$ in digital mimic filter is still $2 \times \mathrm{T}$. The fundamental phasor computation and the ninth harmonic phasor computation under this scenario are demonstrated in Figs. 3 and 4. The results show that the accuracy of phasor computation by EDFT is not affected by the uncertainty of time constant, and the accuracy of phasor computation by DFT-mimic filter configuration suffers from the uncertainty of time constant.

\section{Performance Evaluation of FAult DETECTOR/LOCATOR AND ARCING FAULT DisCRIMINATOR}

\section{A. Simulation System}

The proposed technique is evaluated by considerable computer simulation cases simulated by the Matlab/Power system Blockset simulator [8]. The simulation system is shown in Fig. 5. Transmission lines $\mathrm{T}_{3}$ are the lines to be protected and the PMUs are equipped at BUS S and BUS R to measure the synchronized voltage and current phasors.

The parameters used in the simulation system are listed in Table I. All of the transmission lines are modeled by the distributed parameter model. The voltage sources and source impedances shown in Fig. 5 are Thevenin's equivalences to represent the networks outside BUS A, BUS B, and BUS C.

In this work, the data are sampled at a sampling rate of $3.84 \mathrm{kHz}$ (i.e., 64 sample points per cycle). The length of the moving window is 66 sample points (about $18 \mathrm{~ms}$ ) for computing phasors by EDFT. The faults occur at $54 \mathrm{~ms}$ after the start of simulation. For evaluating the proposed technique, various simulation cases including different fault kinds (arcing and permanent faults), fault types (single-phase ground faults,

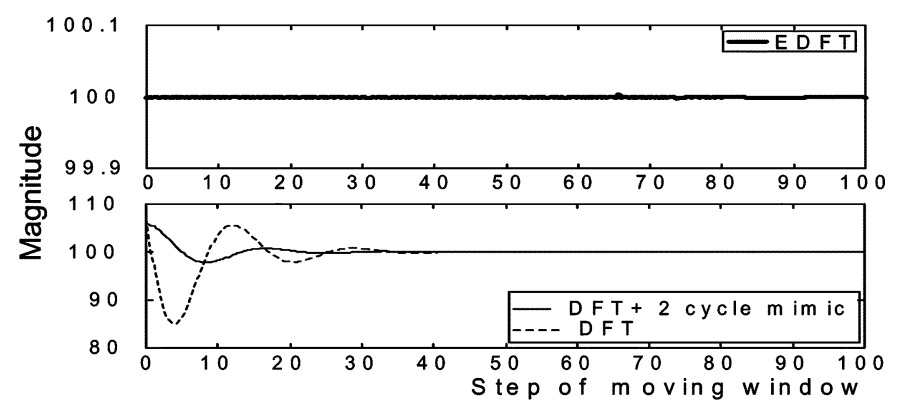

Fig. 3. Computations of the fundamental phasor $\left(\tau_{s}=0.5 \times T ; \tau_{m}=2 \times T\right)$.

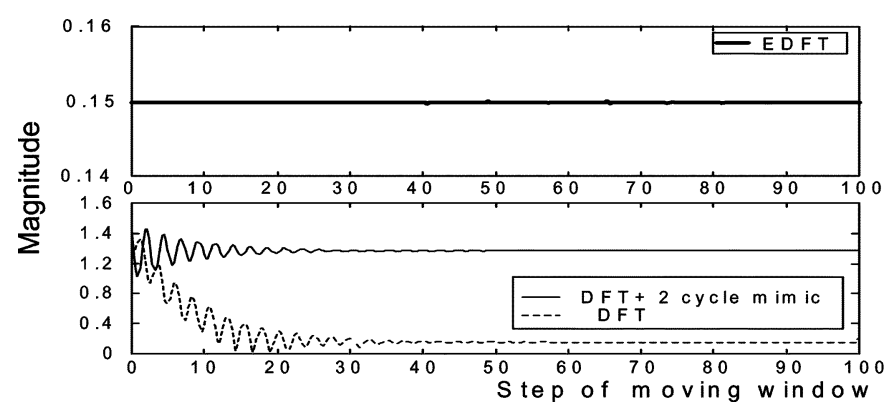

Fig. 4. Computations of the ninth harmonic phasor $\left(\tau_{s}=0.5 \times T ; \tau_{m}=\right.$ $2 \times T)$.

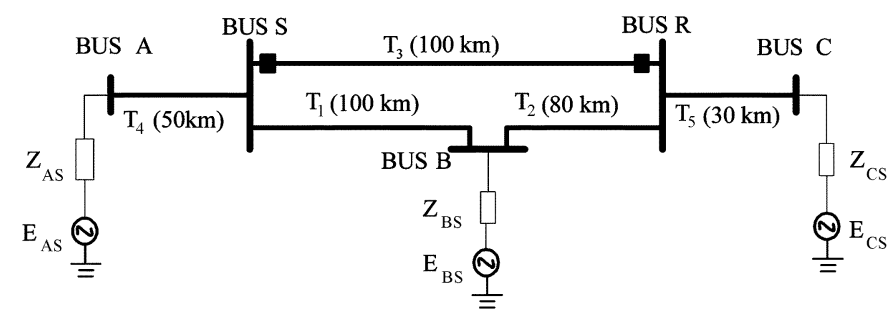

Fig. 5. Simulation system.

TABLE I

PARAMETERS OF SIMULATION SYSTEM

\begin{tabular}{|c|c|c|c|c|}
\hline System voltage: & $345(\mathrm{kV})$ & ; System & uency: & $60(\mathrm{~Hz})$ \\
\hline $\mathrm{E}_{\mathrm{AS}}=1.0 \angle 0^{\circ} \mathrm{p} . \mathrm{u}$ & $\mathrm{E}_{\mathrm{BS}}=$ & $\angle-10^{\circ}$ p.u & $\mathrm{E}_{\mathrm{CS}}=$ & $1.0 \angle-20^{\circ}$ p.u \\
\hline Thevenin's equivalence & & & & \\
\hline $\mathrm{Z}_{\mathrm{AS}, 0}=2.738+\mathrm{j} 10(\mathrm{~S}$ & & & $=0.24+\mathrm{j}$ & $5.71(\Omega)$ \\
\hline $\mathrm{Z}_{\mathrm{BS}, 0}=1.785+\mathrm{j} 7.54$ & $(\Omega)$ & & $=0.42+\mathrm{j}$ & $5.95(\Omega)$ \\
\hline $\mathrm{Z}_{\mathrm{CS}, 0}=0.833+\mathrm{j} 5.18$ & & & $=0.24+\mathrm{j}$ & $6.19(\Omega)$ \\
\hline Transmission lines para & neters: & & & \\
\hline $\mathrm{R}_{0}=0.398(\Omega / \mathrm{km})$ & & & $0.075(\Omega$ & $2 / \mathrm{km})$ \\
\hline $\mathrm{L}_{0}=3.1 \times 10 \mathrm{e}-3(\mathrm{H} / 1$ & & & $.937 \times 10$ & $\mathrm{e}-3(\mathrm{H} / \mathrm{km})$ \\
\hline $\mathrm{C}_{0}=21 \times 10 \mathrm{e}-9(\mathrm{~F} / \mathrm{k}$ & & & $21 \times 10 \mathrm{e}$ & $-9(\mathrm{~F} / \mathrm{km})$ \\
\hline
\end{tabular}

symmetrical faults...etc.), amplitudes of arc voltage, fault locations, fault resistance, injection of external harmonic source, and test examples with more realistic arc model are demonstrated systematically. In all of the simulation cases, the third, fifth, seventh, and ninth harmonic phasors are utilized to estimate the amplitude of arc voltage for arcing fault discrimination. 

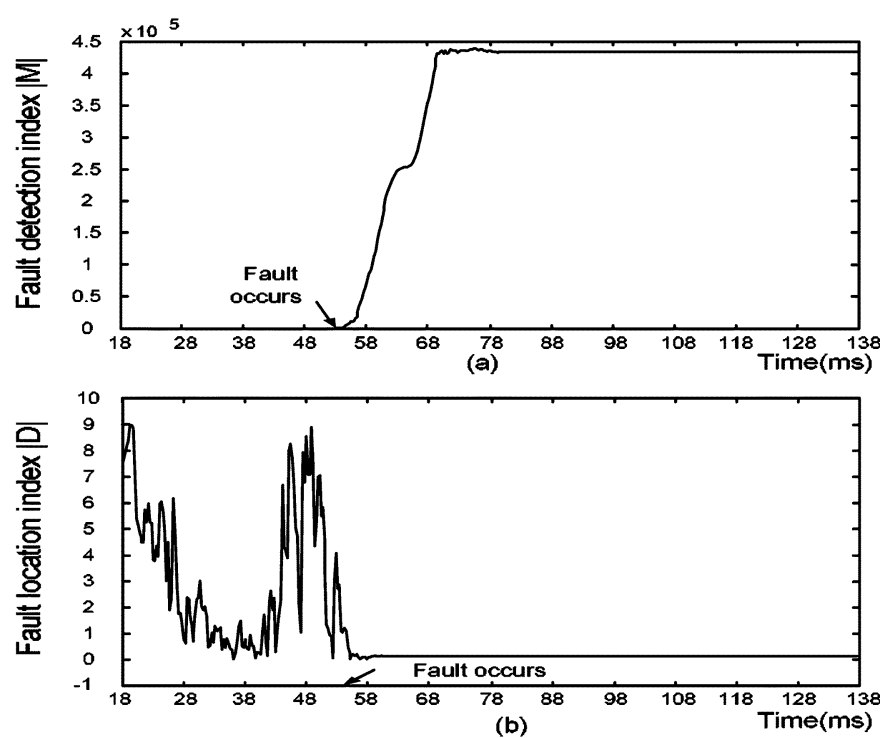

Fig. 6. Typical variations of $|M|$ and $|D|$ when an in-zone fault occurs.
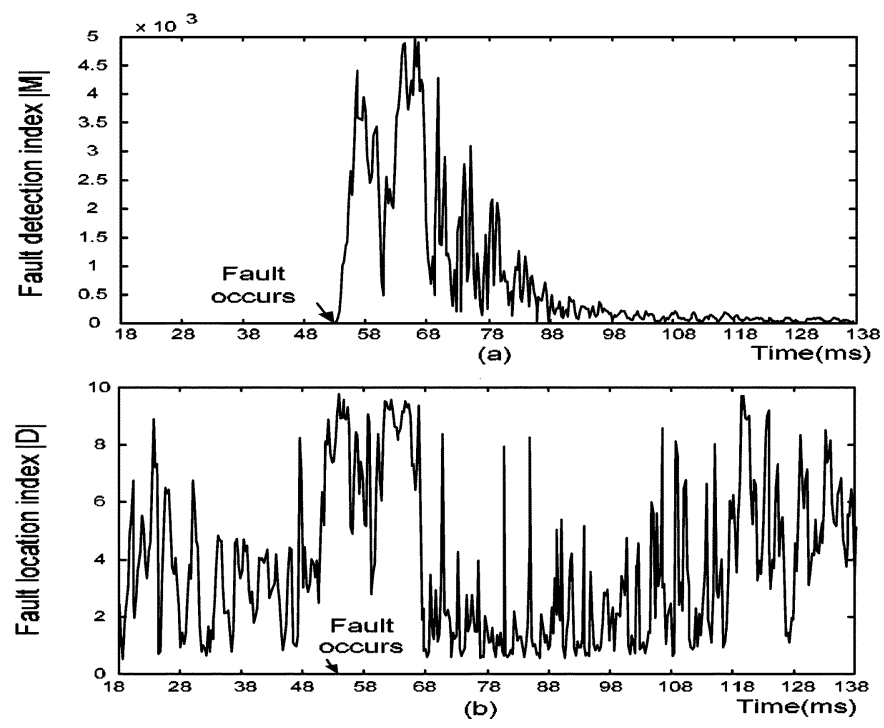

Fig. 7. Typical variations of $|M|$ and $|D|$ when an out-of-zone fault occurs.

\section{B. Determination of Threshold Values}

For determining the threshold value $|D|_{\text {th }}$ and $|M|_{\text {th }}$, the behaviors of $|M|$ and $|D|$ for both arcing and permanent faults are obtained by means of the observation. Fig. 6 shows the typical variations of fault detection index $|M|$ and fault location index $|D|$ when an in-zone fault occurs. In this in-zone fault case, the fault type is an a-phase arcing ground fault, the fault resistance is $1 \Omega$, the amplitude of arc voltage is $1.5 \mathrm{kV}$, and the fault location is 0.1 p.u. away from BUS R. The $|M|$ is equal to zero under prefault condition and will increase rapidly as soon as a fault occurs. And the $|D|$ is an unknown under prefault condition and will converge fast to a value between 0 and 1 when an in-zone fault occurs. Fig. 7 shows the typical variations of $|M|$ and $|D|$ when an out-of-zone fault occurs. In this out-of-zone fault case, the fault type is an a-phase arcing ground fault, the fault resistance is $1 \Omega$, the amplitude of arc voltage is $1.5 \mathrm{kV}$, and the fault location is $40 \mathrm{~km}$ away from BUS S (within $\mathrm{T}_{4}$ ). The

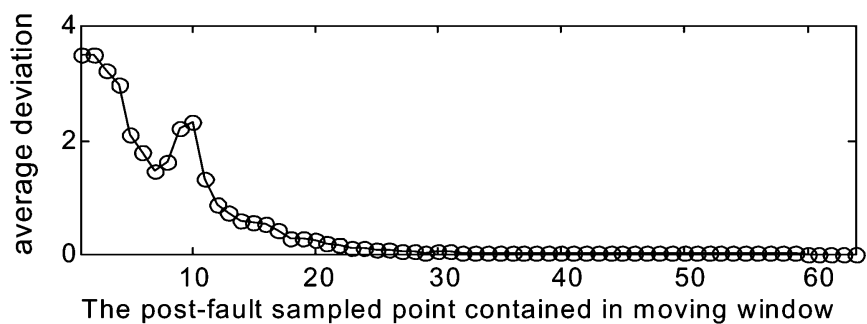

Fig. 8. Average deviation of the average fault location indexes.

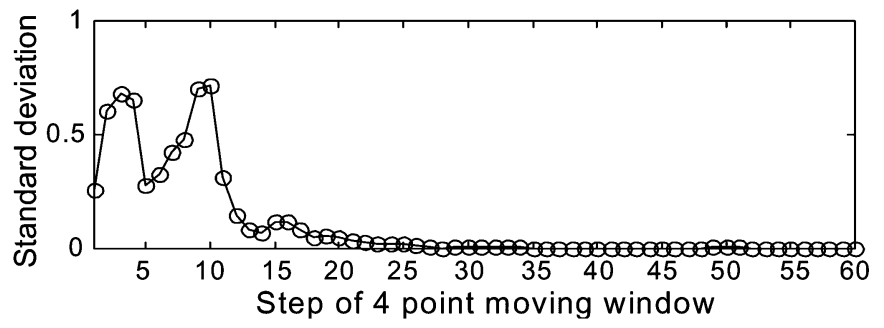

Fig. 9. Standard deviation of the average deviations.

$|M|$ also increases rapidly as soon as a fault occurs. And the $|D|$ is an unknown through pre and postfault conditions. Moreover, the variations of $|M|$ and $|D|$ for permanent faults are similar to those for arcing faults. Based on the observation on the variation of $|D|$, the $|D|_{\text {th }}$ can be determined for 462 in-zone fault simulation cases and 278 out-of-zone fault simulation cases by the following steps.

Step 1) Set $\mathrm{n}$ as the postfault sampled points contained in a moving window. For a specific n, compute the average value of the fault location indexes for every simulated fault location. Then, repeat above for $\mathrm{n}=1$ to 64 .

Step 2) For a specific n, compute the average deviation of the average values. The deviation for a specific fault location is the difference between the average value and actual fault location. Then, repeat above for $n=1$ to 64 . Step 3) Compute sequentially the standard deviation of these average deviations in a four-point moving window. Step 4) Select sequentially a datum from the beginning to the end of the data obtained by Step 3 as the candidate for threshold value $|D|_{\mathrm{th}}$. Then, test the selected datum to all of the in-zone fault cases and out-of-zone fault cases. When the desirable trip speed, dependability, and security are achieved, the selected one is chosen as the threshold value $|D|_{\text {th }}$.

The result after performing steps 1 and 2 are shown in Fig. 8. And the result after performing step 3 is shown in Fig. 9. Finally, the value 0.0268 which locate at the 22th step in Fig. 9 is selected as the threshold value $|D|_{\mathrm{th}}$. Furthermore, the threshold value $|M|_{\mathrm{th}}$ is chosen as 3.118 for fault detection. This is determined by the following equation:

$$
|M|_{\mathrm{th}}=\mu+k \times \sigma
$$

where $\mu$ and $\sigma$ are the mean value and standard deviation of 32 consecutive $|M|$ under prefault conditions. And $k$ is chosen as 3 in this study. 


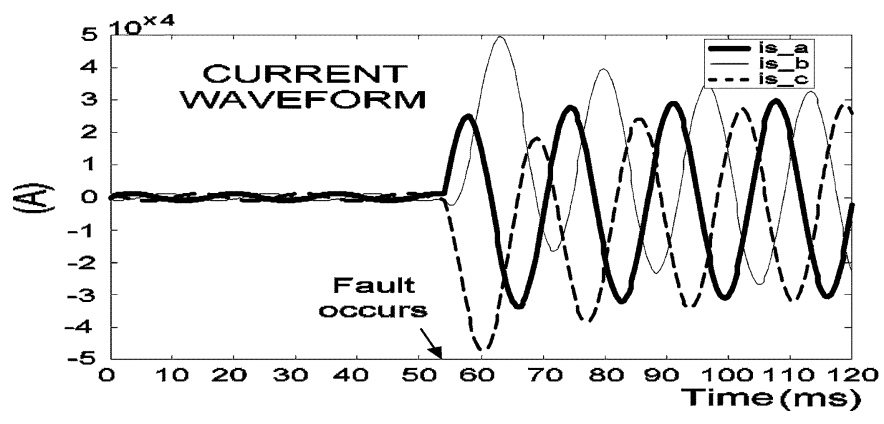

Fig. 10. Current waveforms (symmetrical arcing fault).

Similarly, the threshold value $V_{\text {th }}$ for the arcing fault discrimination can be determined by (2). Instead, the $\mu$ and $\sigma$ denote the mean value and standard deviation of the estimated amplitudes for 154 in-zone permanent fault cases, respectively. Since the estimated amplitudes converge well within four cycles after fault inception, we use the estimated amplitudes at the fourth cycle after fault inception to determine $V_{\mathrm{th}}$. The statistic data are: the mean value is $8.12 \mathrm{~V}$ and the standard deviation is $4.1 \mathrm{~V}$. Thus, the $V_{\text {th }}$ is chosen as $20.42 \mathrm{~V}$. Certainly, the $k$ in (2) can be chosen as a larger value than 3 for discriminating between arcing and permanent faults faster.

\section{Simulation Results of the Proposed Technique}

1) Performance Evaluation of the Overall Protection System: In this subsection, the detailed simulation results for three fault types (i.e., symmetrical faults, single-phase ground faults, and double-phase faults are demonstrated).

a) Symmetrical faults: Two simulation cases of symmetrical fault-one is an arcing fault case and the other is a permanent fault case-are demonstrated herein. Both of the arcing and permanent faults occur at 0.9 p.u. away from BUS $\mathrm{R}$. The amplitude of arc voltage is set as $3 \mathrm{kV}$. Fig. 10 shows the current waveforms measured at BUS S for the arcing fault case. It is obvious that the postfault b-phase current denoted by is_b contains large exponentially decaying dc offset after fault inception. Parts of the phasor computations of b-phase current are demonstrated in Fig. 11. Both phasor computations by EDFT and DFT are shown simultaneously for comparison. The fundamental, third harmonic, fifth harmonic, and ninth harmonic phasors are demonstrated in Fig. 11(a)-(d), respectively. Taking observation on Fig. 11, it is obvious that the EDFT eliminates the error caused by exponentially decaying dc offset and increases the convergence speed. Fig. 12 shows the fault location index and trip signal for the arcing fault case. Using the determined threshold value $|D|_{\text {th }}$, the trip signal is launched at $9.3 \mathrm{~ms}$ after fault inception. Fig. 13 (a) and (b) shows the estimated amplitudes of arc voltage for the arcing fault and permanent fault cases, respectively. In Fig. 13(a), the estimated voltage converges fast to the set value after fault inception. In Fig. 13(b), the estimated voltage converges fast to zero after fault inception. Using the determined $V_{\mathrm{th}}$, the kinds of faults can be discriminated easily within four cycles after fault inception.
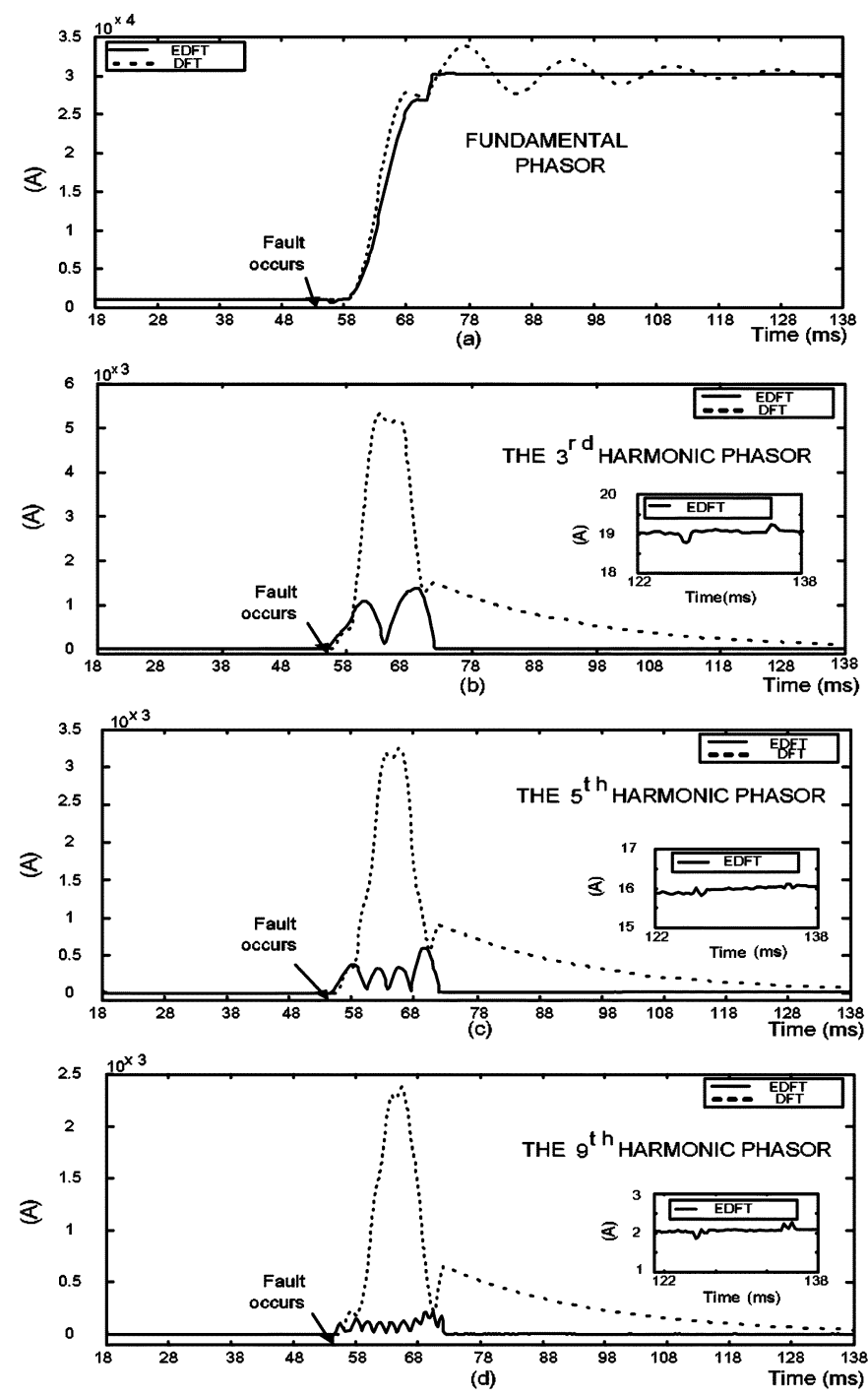

Fig. 11. Phasor computations of the b-phase current (symmetrical arcing fault).

b) Single-phase ground faults: Two simulation cases of a-phase ground fault, one is an arcing fault case and the other is a permanent fault case, are demonstrated in this subsection. Both of the faults occur at 0.9 p.u away from BUS R. In the arcing fault case, the amplitude of arc voltage and fault resistance are set as $1.5 \mathrm{kV}$ and $1 \Omega$, respectively. The fault resistance is set as $1 \Omega$ in the permanent fault case. Fig. 14 shows the fault location index and trip signal for the arcing fault case. The trip signal is launched at $10.2 \mathrm{~ms}$ after fault inception. Fig. 15(a) and (b) shows the estimated voltages for the arcing fault and permanent fault cases, respectively. In Fig. 15(a), the estimated amplitude converges fast to the set value. In Fig. 15(b), the estimated amplitude converges fast to zero after fault inception. Using the determined $V_{\mathrm{th}}$, the kinds of faults can be discriminated within four cycles after fault inception.

c) Phase-to-phase faults: Two simulation cases of bc-phase fault, one is an arcing fault and the other is a permanent fault, are demonstrated in this subsection. The faults occur at 0.35 p.u. away from BUS R. In the arcing fault case, the 


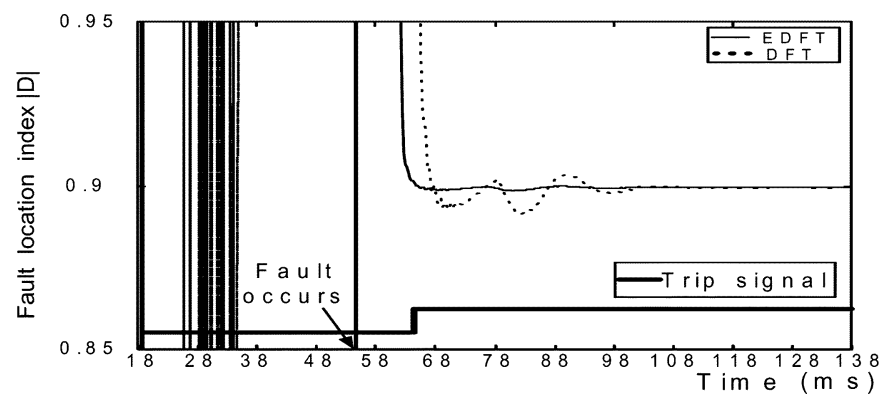

Fig. 12. Fault location index and trip signal (symmetrical arcing fault).
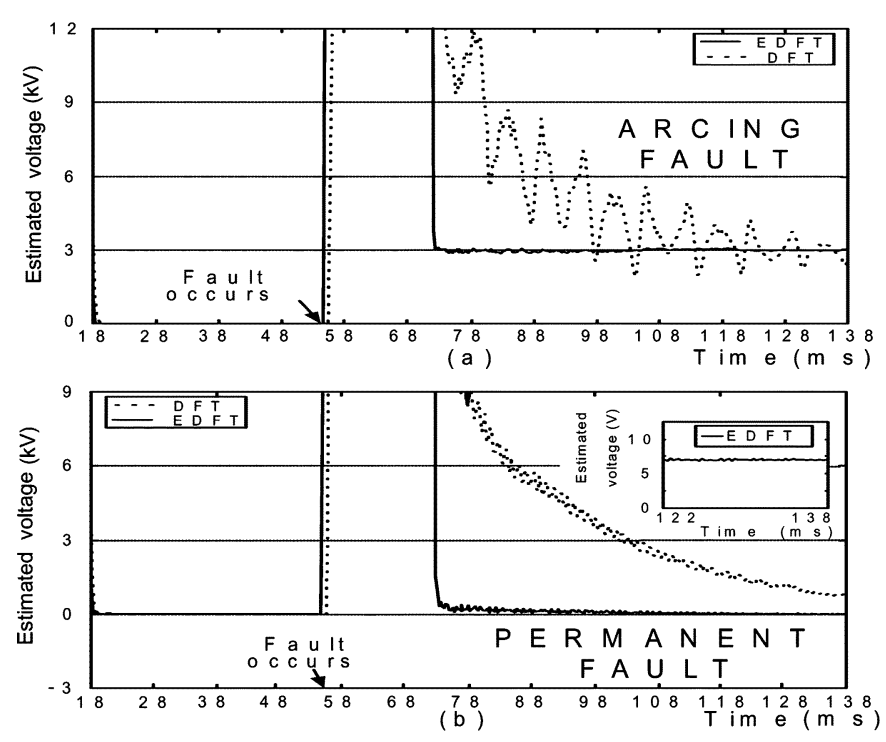

Fig. 13. Estimated amplitudes of arc voltage (symmetrical faults).

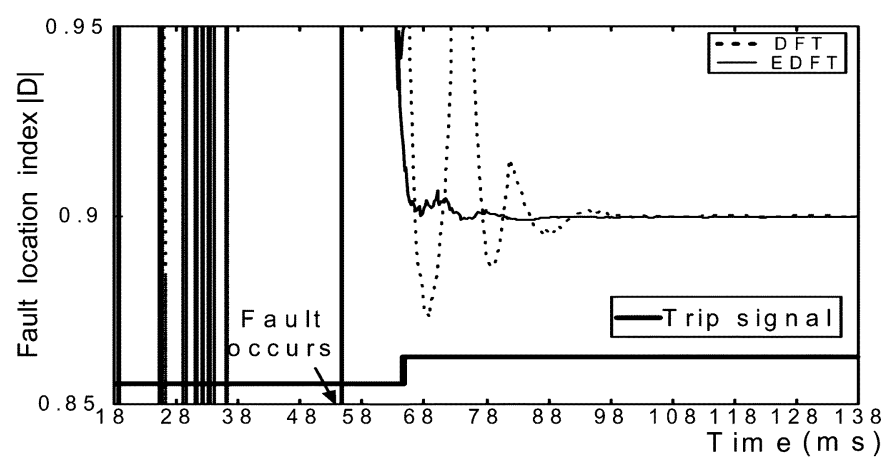

Fig. 14. Fault location index and trip signal (a-phase arcing ground fault).

amplitude of arc voltage is set as $2 \mathrm{kV}$. Fig. 16 shows the fault location index and trip signal for an arcing fault case. The trip signal is launched at $6.28 \mathrm{~ms}$ after fault inception. Fig. 17(a) and (b) shows the estimated voltages for the arcing fault and permanent fault cases, respectively. Obviously, the kinds of faults can be discriminated easily within four cycles after fault inception.

2) Performance Evaluation of the Fault Detector/Locator: To evaluate the performance of proposed fault detection/location technique, various computer simulations including different fault kinds, amplitudes of arc voltage (from 0 to $5 \mathrm{kV}$ ), fault types, fault locations (from 0.01 to 0.99 p.u.), fault resistance
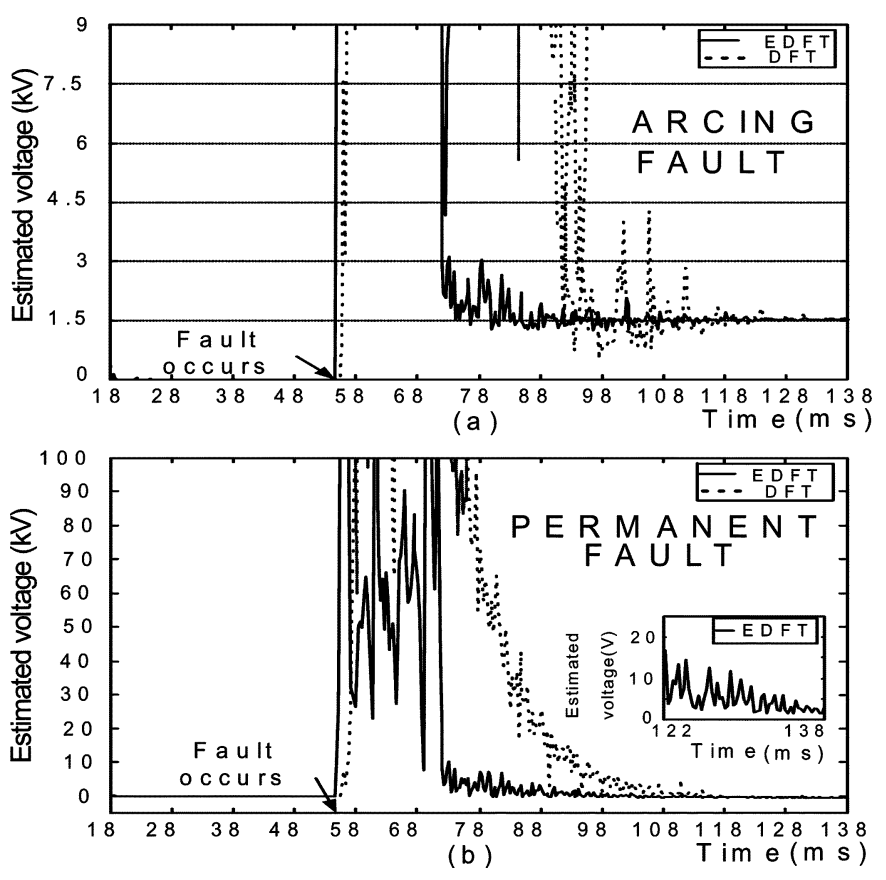

Fig. 15. Estimated amplitudes of arc voltage (a-phase ground faults).

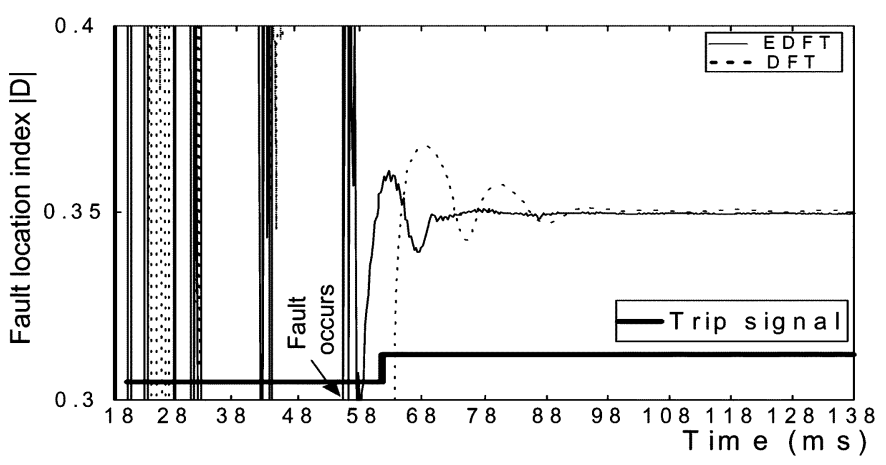

Fig. 16. Fault location index and trip signal (bc-phase arcing fault).

(from 1 to $100 \Omega$ ), fault angles, and loading conditions are performed. The performance of the fault detector is evaluated by the trip time. And the trip time is defined as

Trip time $=$ the time when the trip signal is launched

- the time when the fault occurs.

The performance of fault locator is evaluated by the error of fault location at the fourth cycle after fault inception. And it is defined as

$$
\begin{aligned}
& \operatorname{Error}(\%) \\
& =\frac{\mid \text { Actual fault location }- \text { Computed fault location } \mid}{\text { The length of protected lines }} . \\
& \quad \times 100
\end{aligned}
$$

For 462 in-zone simulation cases, the trip time achieved can be up to $3.25 \mathrm{~ms}$ and does not exceed $12 \mathrm{~ms}$. The average trip time is about $8 \mathrm{~ms}$ and the dependability is $100 \%$. For 278 out-of-zone fault cases, the security is $100 \%$. Furthermore, the fault location error achieved can be up to $0.002 \%$ and does not exceed $0.45 \%$. 

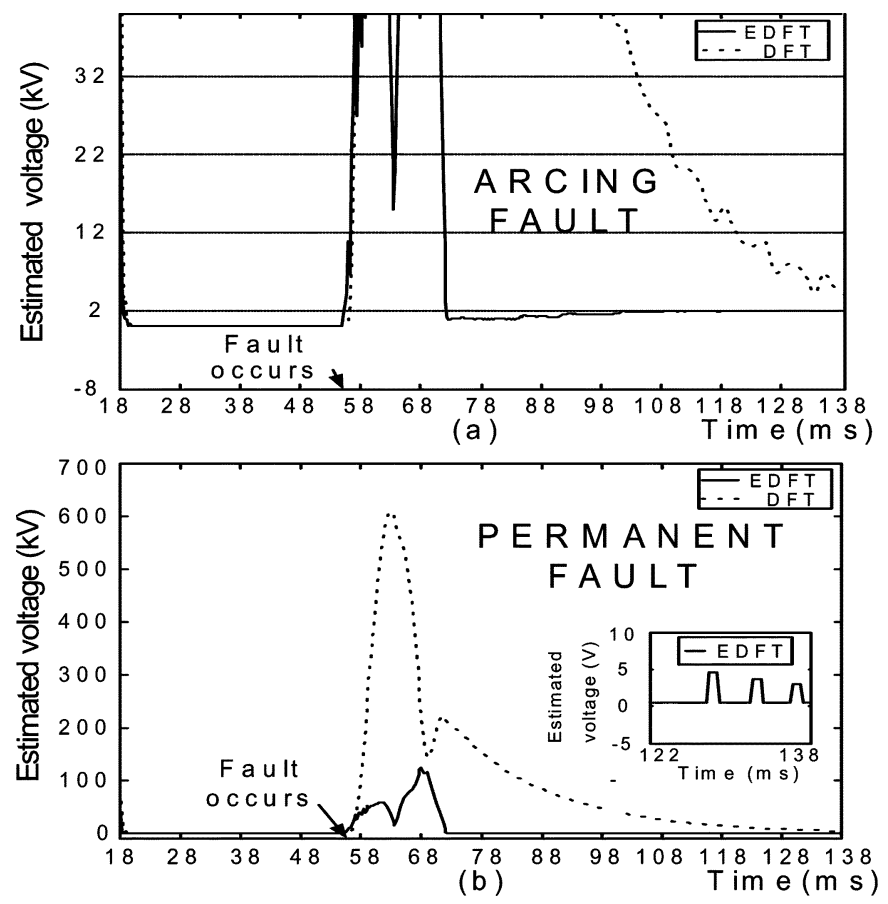

Fig. 17. Estimated amplitudes of arc voltage (bc-phase faults).

In order to investigate the effect of arc model on the proposed fault detection/location algorithm, we evaluate the proposed algorithm to the more realistic arc model represented by (1) in Part I of this two-paper set. In this simulation case, the stationary arc conductance is given by Kizilcay's model as the following [9], [10]:

$$
G=\frac{\left|\mathrm{i}_{\text {arc }}\right|}{\left(U+R_{\text {arc }} \times\left|i_{\text {arc }}\right|\right)}
$$

where $i_{\text {arc }}$ is the arc current; $U$ is the constant voltage parameter, and $R_{\text {arc }}$ is the resistive component.

The fault type is an a-phase arcing ground fault with fault resistant $=10 \Omega$ and fault location $=0.4 \mathrm{p} . \mathrm{u}$. away from BUS R. The parameters for (3) are $\mathrm{U}=3 \mathrm{kV}$, $R_{\text {arc }}=0.0137 \Omega$, and $\tau=0.625 \mathrm{~ms}$. Fig. 18 shows the simulation result. The trip signal is launched at $7.18 \mathrm{~ms}$ after fault inception and the fault location error is $0.078 \%$. The simulation result shows that the proposed fault detector/locator also gives the good result when the more realistic arc model is applied.

3) Performance Evaluation of Arcing Fault Discriminator:

a) Different amplitudes of arc voltage: To evaluate the effect of different amplitudes of arc voltage on the proposed technique, four amplitudes- $0,1.5,3$, and $4.5 \mathrm{kV}$-are tested. Fig. 19 shows the estimated voltages when a-phase arcing ground faults with fault resistance $=10 \Omega$ occur at 0.3 p.u. away from BUS R. The $x$-axis shows the time after fault inception. Fig. 19 shows clearly that different amplitudes of arc voltage do not affect the proposed algorithm. The estimated voltages converge fast to the correct value after fault inception. Moreover, the tests for other amplitudes of arc voltage also give good results as well.

b) Different fault locations: To investigate the impact of different fault locations on the estimation of amplitude of arc

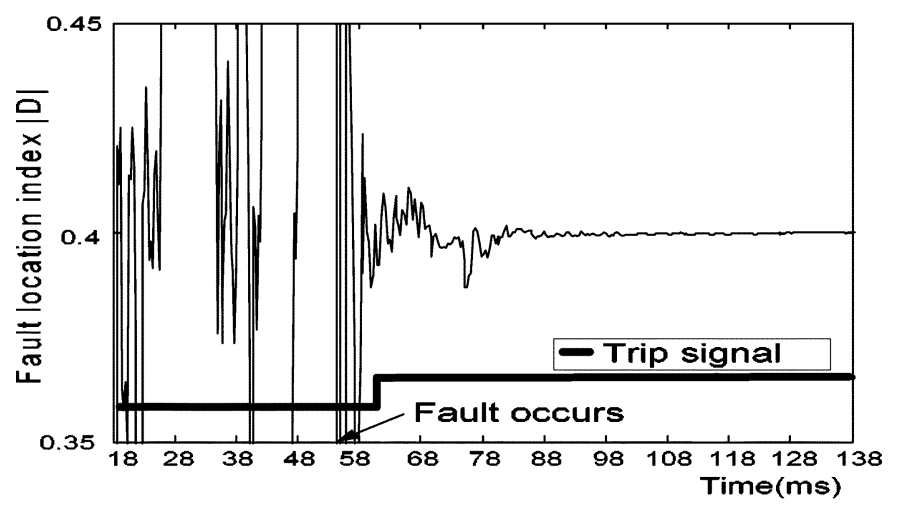

Fig. 18. Fault location index and trip signal (test with more realistic arc model).

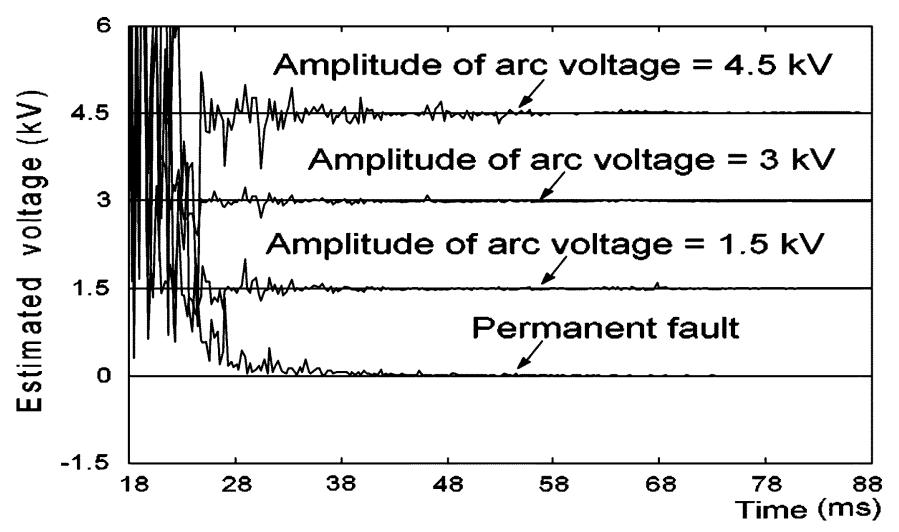

Fig. 19. Estimated voltages under different amplitudes of arc voltage.

voltage, three a-phase ground faults with fault resistance $=$ $1 \Omega$ and the amplitude of arc voltage $=1.5 \mathrm{kV}$ are tested. The tested fault locations are $0.2,0.5$, and 0.8 p.u. away from BUS R, respectively. Fig. 20(a)-(c) shows the simulation results. The $x$-axis shows the time after fault inception. Fig. 20 demonstrates clearly that the proposed algorithm is not affected by different fault locations. The estimated voltages converge fast to the correct value after fault inception. Furthermore, the tests for other fault locations also give good results as well.

c) Different fault resistance: Fault resistance is one of the major factors that affect the algorithm for arcing fault discrimination based on one terminal measurement approach [11], [12]. To investigate the effect of different fault resistance on the proposed algorithm, we vary the fault resistance deliberately from 1 to $100 \Omega$ in the a-phase ground faults. In these simulation cases, the amplitude of arc voltage is set as $1.5 \mathrm{kV}$ and fault location is set as 0.7 p.u. away from BUS R. Fig. 21(a)-(c) shows the estimated voltages for the fault resistance $=1 \Omega, 10 \Omega$ and $100 \Omega$, respectively. The $\mathrm{x}$-axis shows the time after fault inception. It is evident that, for different fault resistance, the estimated voltages converge fast to the correct value after fault inception. Moreover, the tests for other fault resistances also give good results as well.

d) Impact of external harmonic source: To investigate the impact of external harmonic source on the proposed arcing fault discriminator, the external third and fifth harmonic currents are artificially injected from BUS S in an unrealistic amount of 


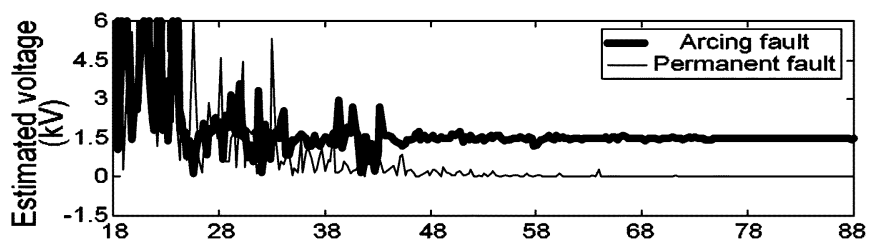

(a)

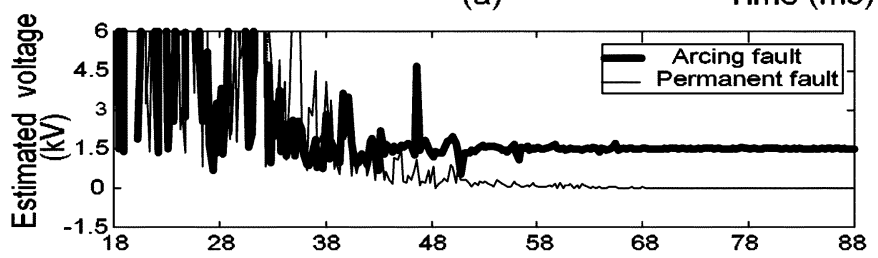

(b)

Time (ms)

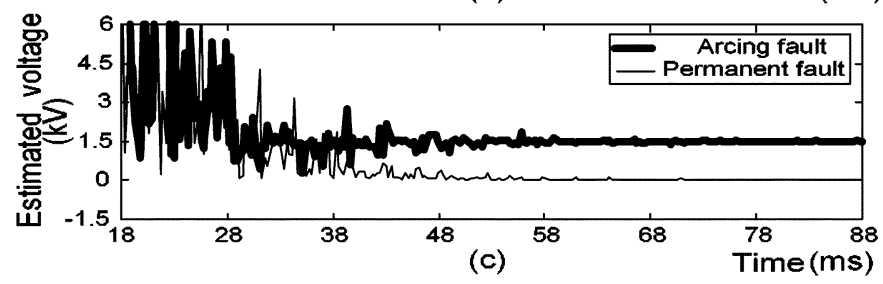

Fig. 20. Estimated voltages under different fault locations. (a) Fault location $=0.2$ (p.u.); (b) fault location $=0.5$ (p.u.); (c) fault location $=0.8$ (p.u.).

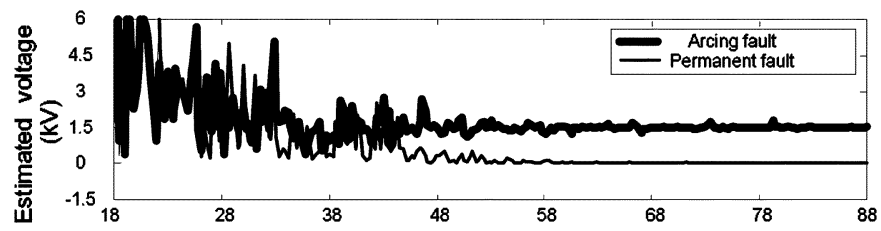

(a)

Time (ms)
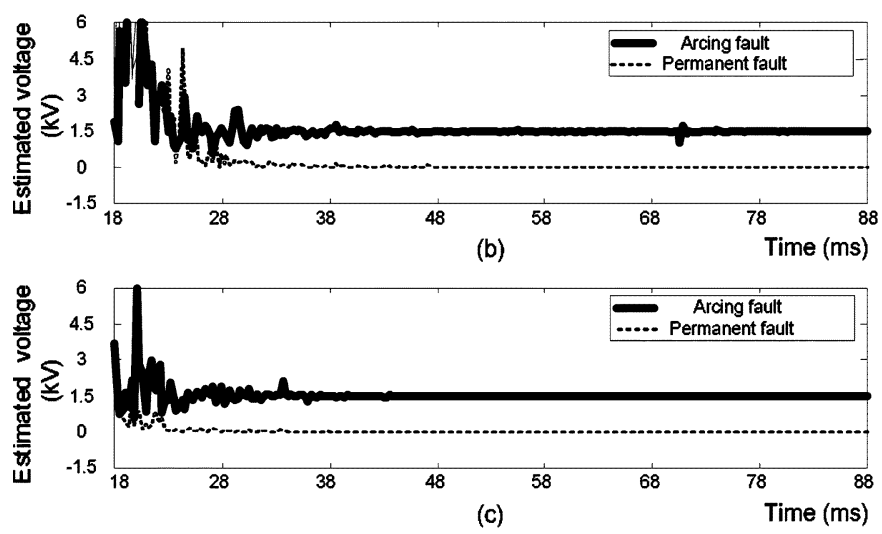

Fig. 21. Estimated voltages under different fault resistance. (a) Fault resistance $=1 \Omega$; (b) fault resistance $=10 \Omega$; (c) fault resistance $=100 \Omega$.

0.5 and 0.2 p.u of prefault loading, respectively. In these simulation cases, a-phase ground faults occur at 0.75 p.u. away from BUS R with fault resistance $=5 \Omega$ and the amplitude of arc voltage $=2 \mathrm{kV}$. Fig. 22(a) and (b) shows the a-phase current waveform measured at BUS $\mathrm{S}$ for the arcing fault case and the estimated voltages under injection of the external third harmonic source, respectively. Fig. 23(a) and (b) shows the magnitude of the a-phase fifth harmonic voltage phasor measured at BUS S for the arcing fault case and the estimated voltages under injection of the external fifth harmonic source, respectively. The simulation results reveal that the accuracy and convergence speed
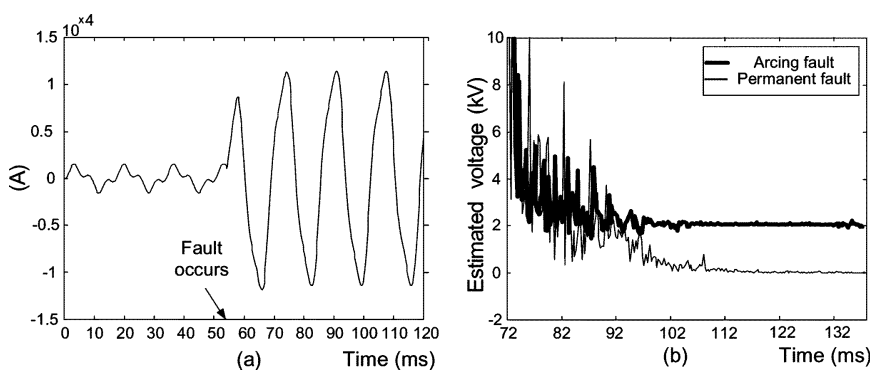

Fig. 22. Simulation cases under injection of the third harmonic source.
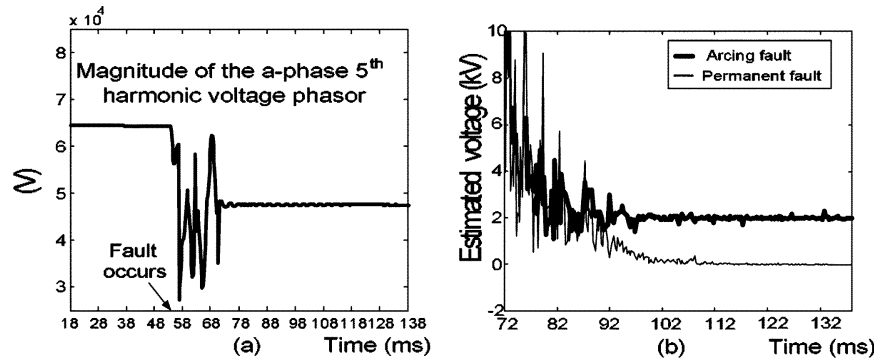

Fig. 23. Simulation cases under injection of the fifth harmonic source.

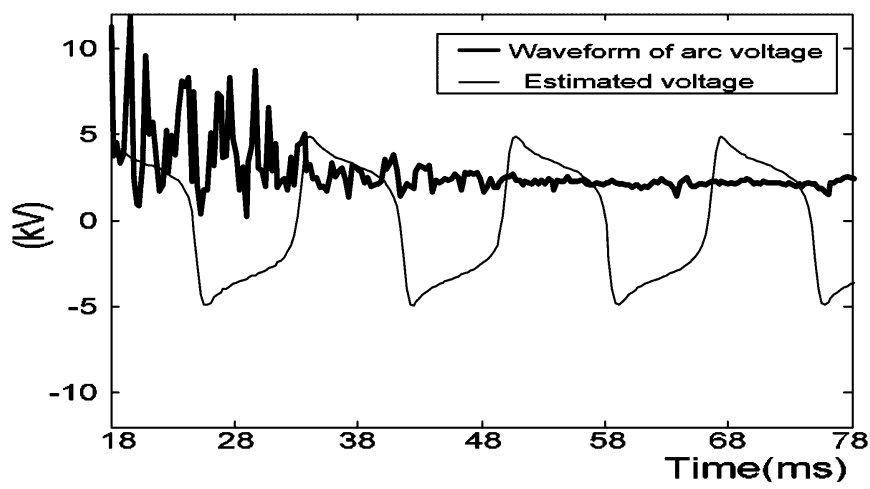

Fig. 24. Estimated voltage for a more realistic arc model.

for estimated voltages are almost not affected by external harmonic current source and the steady harmonic level in network voltage. Furthermore, the tests with injection of the seventh and ninth harmonic sources give good results too.

e) Test with more realistic arc model: Since the square wave in phase with arc current is only an approximation of the waveform of the real arc voltage, we evaluate deliberately the proposed algorithm to the more realistic arc model represented by (1) in Part I of this two-paper set. In this simulation case, the fault type is an a-phase arcing ground fault with fault resistant $=10 \Omega$ and fault location $=0.8$ p.u. The stationary arc conductance is given by Kizilcay's model in (3) with parameters $3 \mathrm{kV}, R_{\mathrm{arc}}=0.0137 \Omega$, and $0.625 \mathrm{~ms}$. Fig. 24 shows the simulation result. The $\mathrm{x}$-axis shows the time after fault inception. The simulation result shows that the estimated voltage converges to about half of the maximum value of arc voltage. It is easy to identify the fault as an arcing fault.

f) Test using the real-life measured data: On April 19, 2002 , an a-phase arcing ground fault occurred on the $345-\mathrm{kV}$ transmission lines between Lungchi substation and the third nuclear power plant substation on the Taiwan power system. The 


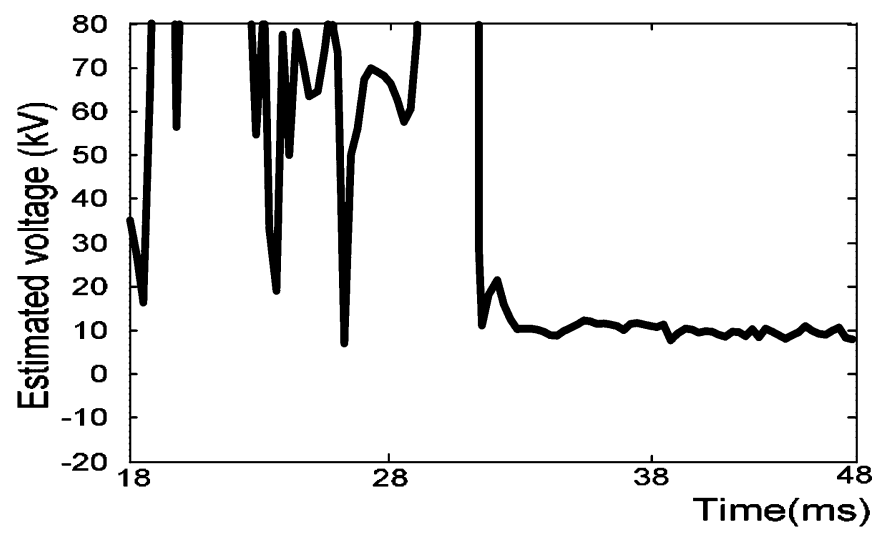

Fig. 25. Estimated voltage for a real-life arcing fault case.

fault was caused by fire under a pylon. The length of faulted lines is $127.3 \mathrm{~km}$ and the fault occurred at about $9 \mathrm{~km}$ away from Lungchi substation. For this real-life arcing fault case, the error of fault location is $0.76 \%$ by the proposed fault locator. And the estimated voltage is shown in Fig. 25 . The $x$-axis shows the time after fault inception. The estimated voltage converges to about $10 \mathrm{kV}$. It is easy to identify the fault as an arcing fault.

\section{CONCLUSION}

In this two-paper set, a multifunction transmission-line protection technique based on PMUs is presented. Through a great number of computer simulations, the excellent performance of the proposed technique has been proven. The presented EDFT algorithm eliminates the error caused by exponentially decaying dc offset on the phasor computation. It forms the basis for highspeed phasor-based digital relays. For the proposed fault detector, the trip time achieved can be up to $3.25 \mathrm{~ms}$ and the average value of trip times is about $8 \mathrm{~ms}$ for both permanent and arcing faults on transmission lines. For the proposed fault locator, the accuracy can be up to $99.99 \%$ and the error does not exceed $0.45 \%$ for both permanent and arcing faults. Moreover, the proposed acing fault discriminator can discriminate between arcing and permanent faults within four cycles after fault inception. It has been proven to be an effective tool to block reclosing on permanent faults in the computer simulations. Finally, a test case using real-life measured data proves the feasibility of the proposed technique.

\section{REFERENCES}

[1] A. G. Phadke and J. S. Thorp, Computer Relaying for Power System. New York: Wiley, 1988.

[2] A. T. Johns and S. K. Salma, Digital Protection for Power System. Stevenage, U.K.: Peregrinus, 1995.

[3] P. M. Anderson, Power System Protection. New York: McGraw-Hill, 1999.

[4] J.-A. Jiang, J.-Z. Yang, Y.-H. Lin, C.-W. Liu, and J.-C. Ma, “An adaptive PMU based fault detection/location technique for transmission lines: Part I: theory and algorithms," IEEE Trans. Power Delivery, vol. 15, pp. 486-493, Apr. 2000.

[5] J.-A. Jiang, Y.-H. Lin, J.-Z. Yang, T.-M. Too, and C.-W. Liu, “An adaptive PMU based fault detection/location technique for transmission lines: Part II: PMU implementation and performance evaluations," IEEE Trans. Power Delivery, vol. 15, pp. 1136-1146, Oct. 2000.
[6] C.-S. Chen, C.-W. Liu, and J.-A. Jiang, "A new adaptive PMU based protection scheme for transposed/untranspoed parallel transmission lines," IEEE Trans. Power Delivery, vol. 17, pp. 395-404, Apr. 2002.

[7] G. Benmouyal, "Removal of dc-offset in current waveforms using digital mimic filter," IEEE Trans. Power Delivery, vol. 10, pp. 624-630, Apr. 1995.

[8] Power System Blockset User Guide, TEQSIM Inc., a Sublicense of Hydro-Québec and the Mathworks Inc., 1999.

[9] Y. Goda, M. Iwata, K. Ikeda, and S. I. Tanaka, "Arc voltage characteristics of high current fault arcs in long gaps," IEEE Trans. Power Delivery, vol. 15, pp. 791-795, Apr. 2000.

[10] M. Kizilcay and K. H. Koch, "Numerical fault arc simulation based on power arc test," Eur. Trans. Elect. Power Eng., vol. 4, no. 3, pp. 177-185, May/June 1994.

[11] M. B. Djuric and V. V. Terzija, "A new approach to the arcing faults detection for fast autoreclosure in transmission systems," IEEE Trans. Power Delivery, vol. 10, pp. 1793-1798, Oct. 1995.

[12] M. B. Djuric, Z. M. Radojevic, and V. V. Terzija, "Time domain solution of fault distance estimation and arcing faults detection on overhead lines," IEEE Trans. Power Delivery, vol. 14, pp. 60-67, Jan. 1999.

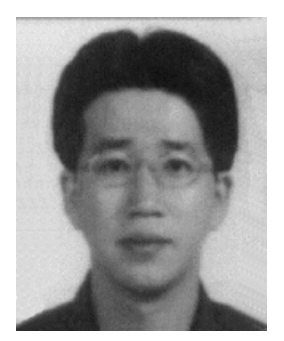

Ying-Hong Lin (S'01-M'03) was born in Taipei, Taiwan, R.O.C., in 1970. He received the B.S. degree in electrical engineering from Taiwan University of Technology and Science, Taipei, in 1995, and the M.S. and Ph.D. degrees in the electrical engineering from National Taiwan University, Taipei, in 1999 and 2002, respectievly.

Currently, he is an Assistant Professor in the Department of Engineering and Management of Advanced Technology, Chang Jung Christian University, Tainan, Taiwan. His research interests are the application of global positioning systems (GPS) and phasor-measurement units (PMUs) in power system protection and very large scale integrated (VLSI) design.

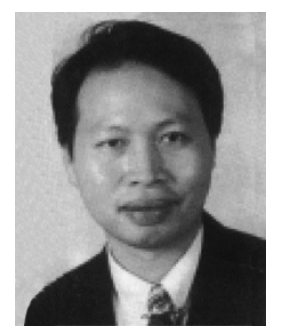

Chih-Wen Liu (S'93-M'96-SM'03) was born in Taiwan, R.O.C., in 1964. He received the B.S. degree in electrical engineering from National Taiwan University, Taipei, in 1987 and the M.S. and Ph.D. degrees in electrical engineering from Cornell University, Ithaca, NY, in 1992 and 1994, respectively.

Currently, he is Professor of electrical engineering with National Taiwan University, where he has been since 1994. His research interests include application of computer technology to power system monitoring, operation, protection, and control. Other research interests include motor control and power electronics.

Dr. Liu is a Reviewer for the IEEE TRANSACTIONS ON POWER SYSTEMS and the IEEE TRANSACTIONS ON POWER DELIVERY.

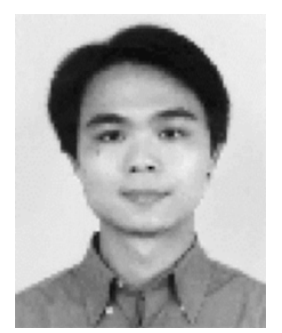

Ching-Shan Chen (S'01) was born in Taichung, Taiwan, R.O.C., in 1976. He received the B.S. degree in electrical engineering from National Taiwan University of Technology and Science, Taipei, in 1998 and the M.S. and Ph.D. degrees in electrical engineering from National Taiwan University, Taipei, in 2000 and 2003, respectively.

His research interests include computer relaying and the application of phasor-measurement units (PMUs) to power system protection. 